BULETINUL INSTITUTULUI POLITEHNIC DIN IAŞI

\section{sciendo}

DOI: 10.2478/BIPMF-2021-0010
(BULLETIN OF THE POLYTECHNIC INSTITUTE FROM IAȘI)

Published by "Gheorghe Asachi" Technical University of Iasi

Volume 67(71), No. 2, 2021

Section Mathematics Theoretical Mechanics. Physics

\title{
FROM CLASSICAL NEWTONIAN PHYLOSOPHY TO SKYRMION - A SHORT HISTORY
}

\author{
NICOLAE MAZILU ${ }^{1,2}$ and CRISTINA-MARCELA RUSU ${ }^{3, *}$ \\ 13707 Dauphin Dr, NE, Canton, OH 44721, USA \\ ${ }^{2}$ Timotei Cipariu, \#1, Focșani, Vrancea, 600004, Romania \\ 3"Gheorghe Asachi” Technical University of Iași, \\ Department of Physics, Iași, Romania
}

Received: May 5, 2021

Accepted for publication: June 10, 2021

\begin{abstract}
This paper presents a short history from Philosophiae Naturalis Principia Matematica of Newton to skyrmions of Skyrme. It is shown that the classical mechanics does not exclude skyrmions (as topologically stable field configuration of a certain class of non-linear sigma models- for example nucleon model). In certain conditions the Newtonian Theory becomes fundamental in building of modern physics theories (as quantum mechanics, fields theories, etc.).
\end{abstract}

Keywords: classical mechanics; skyrmions; quantum mechanics; electrodynamics.

\section{Introduction}

It seems that Skyrme theory unearthed lately in our spirit some old, long forgotten ideas regarding the structure of matter. Indeed, it brings in actuality a peculiar, although quite intuitive manner to conceive the nuclear matter, having ties with the old theory of ether. This manner of conceiving the matter is made possible by the tremendous successes of the process of geometrization of the

*Corresponding author; e-mail: cristina-marcela.rusu@ academic.tuiasi.ro

(C) 2021 Mazilu N. et al. This is an Open access article licensed under the Creative Commons Attribution-Non Commercial - No Dericatives 4.0 International License (CC BY-NV-ND 4.0) 
physical theories, which seems to allow us no alternative, other than the old Newtonian way of natural philosophy. Specifically, that old way relies heavily on analogy, which allows our intellect the transcendence of the daily scale of things, and thus to apply our reason at any other scale of the universe. The geometrization maintains here its role assigned by Newton himself: it shows how to correctly apply the analogy, for there is always a tendency of our spirit to succumb to the glittering temptations of technology. This was the case with the quantization. It is our specific task here to show that the intuitive manner of conception laid down by Newtonian natural philosophy, based on planetary observations, has also intrinsic ties with the very classical model of nuclear atom, as we learn it from the high school curricula.

In spite of the sophisticated mathematical theories explaining one or another from among the multiple aspects of atomic structure, the atom itself is almost exclusively imagined today in the planetary, or nuclear form, first advanced as a reality by Rutherford in 1911. However, even since the emergence of this classical model, contradictory issues popped up, which our spirit duly assigned not to the concept itself, but to the very reality. Leaving aside though the question of how much of such issues should be assigned to the reality per se, and how much to the concept we make out of it, the contradictions they raise indicate by themselves the main physical reason of their existence. This is the close correlation between light and matter, made conceptually possible by the advent of technology. That was the very first point of contradiction, and that is accordingly perpetuated, by education, in our culture, in the form of the intuitively assimilable classical model of atom, still tarnished even today by a bunch of hard to accommodate inconsistencies.

Heinrich Hertz is credited with beginning the present scientific link between matter and light. He is the person who managed describing, and accomplishing actually, the action at a certain range in the shape of electromagnetic signals, therefore ensuring himself a spot in history as the finder of electromagnetic action at range. Because this type of action is scarcely detectable through means of human senses, no claim can be made, for example, that it is accomplished by forces, Hertz depicted it by making use of Maxwell theory of electromagnetic waves, which is solely established on the concept of force as a vector. Beginning relatively early in the previous century, the Hertz dipole, known as as the material structure suitable for making and acquiring electromagnetic waves, was the keystone pertaining to decisions about the material structures related to the formation of light. But what is the meaning of these material structures?

First in line was the electron, revealed by J. J. Thomson in 1897 (Thomson, 1907). On the basis of this discovery, Thomson recomended a matter model. The fact that the matter appears to be in a natural and electrically neutral state was the first thing he assumed. Because the electron holds a negative electric charge, J. J. Thomson postulated the belief that, if the case is 
taken in which matter is constructed of atoms, then such atoms may be imagined as "islands" holding negative charges in a restrained continuum holding positive charges. This represents the so-called "plum pudding" model, describing the atom. Such a depiction pertaining to the structure of matter had, nevertheless, to be deserted at some point, when confronted with experimental facts, which this model of atom could not apparently entertain. Let those facts be shortly analyzed.

It became increasingly obvious around the close of the nineteenth century that the matter is unstable. This is not from the usual, well known, point of view of chemistry but, rather from the old perspective of alchemy: the elements themselves, and, as such, the atoms, turned out to be unstable, and thus evolving in new elements - the ultimate aspiration of alchemists! Specifically, heavier elements were disintegrating towards lighter elements, emitting three forms of radiation named by Ernest Rutherford alpha, beta, and gamma. Alpha and beta radiations proved to be made out of charged particles, due to the fact that they notably responded to electric and magnetic forces. The beta radiation was readily described employing the electrons of J. J. Thomson. The alpha radiation had a positive charge electrically, but it was made up of massive particles that were far heavier than electrons. These particles had the mass of the helium atom, with the difference between those two being their electrical charge, unlike the helium atom, which was neutral. The gamma rays were electrically neutral and had a high penetration rate through matter.

It is worth noting that natural philosophy adopted, perhaps for the first time when it came to structural units of matter, the reasoning commonly used in light experiments. Certainly, the quantitative features of matter units, such as light, are not directly visible to human senses, but rather through the "looking glass" of certain effects caused by these units on other portions of matter, subsequently visible to human senses. Therefore, the experimental works on the radiations alpha, beta and gamma were made in the manner of J. J. Thomson, using high vacuum Crookes tubes, fitted with electrodes, as a means to design electrical deflecting fields. In the case in which the particles did not exhibit an electrical charge, they were merely not deflected, that effect being directly observable.

During suchlike experiments, which were undertaken under the supervision of the Thomson atomic model, Rutherford and Geiger observed an unexpected phenomenon. If a mica slate is placed between the source of alpha radiation and the plate used as a recording medium for the particles, then the intensity of the recorded particles is decreased. This indicates that the entire amount of alpha particles was not able to pass through matter; several particles were stuck inside. A study for the quantitative evaluation of the observed effect in different materials was started and Marsden was tasked with proving that no scattering events exist at varying angles with regard to the direction between the radiation source and the target through experimentation. This could fully 
validate the idea based on the Thomson atom model. The shock was palpable: the alpha particles were dispersed in all directions, including in reverse direction! Most of the particles, but not all, of them went forward.

These discoveries led Rutherford to conclude that the idea of the atom as "plum pudding" isn't entirely true. Far closer to reality is the notion that an atom is mostly void, exhibiting a positive charge condensed in a nucleus and the electrons at long distances from the nucleus. Charles Coulomb had already proven that, like Newton's gravitational force, the force amongst electric charges varies with the distance between them. Logically, and reasonable at the time, to think that electrons move around a positive nucleus in the same way that planets move around the Sun. Thus the nuclear model of atom in physics was born (Rutherford, 1911).

This represented the historical meeting of theoretical physics and astrophysics, at that time a science that required an explanation of the process by which the Sun produces light. This light's spectra revealed remarkable regularities, recognized as spectral series, with bright lines in certain points in the spectrum. The experimental physics had resources available to link the light spectra with chemical elements liberally existing on Earth, especially the hydrogen, which appeared to be the main component in the spectra of celestial bodies. As a result, it was normal to credit the production of light in varied conditions to the hydrogen atom, which certainly is present in the Sun and stars.

Taking into account the fact that light was viewed as an electromagnetic radiation, the entire concept was evident and straightforward to depict physically: the hydrogen atom as envisioned by Rutherford can naturally spawn electromagnetic radiation, due to the fact that its electron exibits perpetual acceleration, and thus it emits radiation much like a Hertz dipole antenna. A Rutherford atom is possible to be thought of as a planar harmonic oscillator, that can then be handled as a Hertz dipole. The radiation is the emitted continuously. It was at this time that it was revealed that the Rutherford atom does not follow classical principles. Obivously, the emission of radiation equates to the one of energy, but this holds true only as long as energy is available. The emission comes to a halt when the energy source is depleted. As a result of the continual emission of light, it will be only natural for the atom to vanish. There was, however, no experimental data to back up this claim.

Niels Bohr discovered the solution by comparing the regularities of the hydrogen spectrum with the mechanical possibilities of the classical planetary model in the energy of the model: it works in such a way that only certain orbits of the electron around nucleus are possible, and these have fixed energy (Bohr, 1913). The light produced by an atom corresponds to some instantaneous transitions of the electron between different orbits, and carries with it the difference of energy of the orbits between which the transition is made. Bohr used the theory, which was novel at the time, that light in such instances is not exactly dense energetically, and can be characterized by an ideal gas of 
particles, according to Einstein (Einstein, 1905), each one of them carrying a quantum of energy. To put it another way, light is released and absorbed at the atomic scale in a form known as a photon (Lewis, 1926).

Even though Bohr's statements saved the day, so to speak, for the atomic model of Rutherford, a drawback remained, and still haunts the science even today: according to our notion of electric charge, an atomic nucleus should be unstable. Not in the way in which, for instance, the Sun or the Earth are unstable, but in a way, we should say, much more severe. While the Sun and the Earth are in a slow continuous change, but still remain confined in a space region which is predictable on all its aspects, according to our notions of electricity the nucleus should break into pieces and scatter instantaneously. In a way, this is indeed the case, as the phenomenon of disintegration, giving the three kinds of radiation mentioned above, plainly shows. However, the instantaneous destruction of the nucleus is not a permanent phenomenon, a daily phenomenon so to speak, as our notion of charge asks, but is only incidental. Thus, if there are sufficient reasons to think that other atoms might have an unstable nucleus, the hydrogen, at least, does not show such behavior. This fact is quite sufficient in order to give us an enigma: how come that the electric repulsion forces do not break and scatter the nucleus apart?

At this point, for the first time since Newton, a wonderful thing happened: a new fundamental force was allowed to enter the game, in exactly the Newtonian manner, i.e. based on experience. First, the neutron was discovered, which was immediately allotted to nucleus as a component of its structure, with no electric charge, but being however capable of developping strong ties with the proton - the positively charged component of the nucleus. Thus, at least in the case of the atoms with the number of mass different from the number of proton charges, theoretical physics conceded to the existence of another type of fundamental forces besides the Newtonian ones. These forces, called nuclear forces for obvious reasons, must be very strong at small distances - therefore inside nucleus - and prevail over any kind of repulsion forces, inversely proportional with the square of the distance between material particles. They were pretty obvious in the existence of alpha particle, but the acceptance of their very existence was apparently sealed once the deuterium was discovered. The first attempt of their explanation was that of Werner Heisenberg in 1932 by quantum mechanical methods (see Brink, 1965 for a review of the story of nuclear forces and English translations of Heisenberg originals).

Heisenberg, as well as Fermi two years later in a first theory of beta decay, calculated the nuclear forces indirectly, through a perturbative term in the Hamiltonian, giving rise to the the so-called "platzwechselintegral" exchange integral. However, this method underestimated the forces by comparison with experimental data. Hideki Yukawa (Yukawa, 1935; see also the reprint in Brink, 1965 part 2, and the collection of reprints Beyer, 1949) was 
the one to notice that, if it is to extend over the nuclear matter the analogy with the classical theories, then we should look a little deeper and a little further into the facts. First of all, if it is to have a quantum there, like in the case of light quantum, then we should start with the D'Alembert equation - the one from which the space-time quantization for light starts - and not with the Schrödinger equation, as everyone started probably at the suggestion of Gamow in a pioneering article (Gamow, 1928; see also the collection Beyer, 1949). This fact would renew the tricky problem of quantum per se, still fresh in the epoch, but Yukawa has a genuine approach, probably inspired by an old problem which certainly has analogous connotations. That was the problem of discrepancy between the apparent uniformity of matter in the universe, as suggested to us by the light of heavens, and the matter compatible with Newton's law of force, approached by Seeliger in an original manner (Seeliger, 1895). As we see it, this is ultimately a mere discrepancy between light and matter. And in spite of the fact that the motion of matter is thought to be here the fundamental ingredient solving the problem, thus delegating the solution to relativistic tools, we still think that the old static philosophy deserves all our attention.

So, in his approach to nuclear forces, Yukawa noticed first the necessity of transforming the D'Alembert wave equation for the nuclear potential depending on coordinates and time inside nucleus, into an equation that can account for the finite nonzero mass of the 'quantum of nuclear force'. And he proceeded in the manner of Seeliger, by adding an exponential factor to the classical wave solution of the D'Alembert equation. Indeed, in terms of differential equations, the Seeliger's procedure was simply to transform the Laplace equation for the potential of Newtonian forces, into a Helmholtz equation, by admitting an exponential solution in coordinates. If the same was to be true for the potential of nuclear forces then, given the relativistic ideas, the procedure had to be applied to the D'Alembert equation. The resulting solution had therefore to satisfy what we call today the Klein-Gordon equation, which entered the history of the theoretical physics in the same year 1926, so fateful for the quantum mechanics (see Pauli, 1980, p. 146ff).

So much for the potential inside the nuclear matter. The equation proposed by Yukawa would admit forces different from the Newtonian ones, appropriate to describe nonzero uniform density of a moving matter, and apparently fit for nuclear matter. It would remain only the problem to materialize the idea that the exchange forces are always accompanied by the kind of events we expect in sudden fragmentation of matter, like the emission of an electron or a positron. And Yukawa had here apparently a fresh idea, again in keeping with the classical theory of forces. Indeed, by his approach, it was quite clear that the issue at stake was that of the density of matter, which from the point of view of classical mechanics was instrumental in the determination of forces in a continuum. However, there is here a hidden trap: if the density determines the potential of forces via Poisson's equation, then at least its 
nonuniformity must be defined itself by some other rules than those of classical mechanics. Otherwise the definition can create a vicious cycle, as it always did. In that case we would have to use a self-consistent theory, possibly of the Thomas-Fermi kind, and this is not always fortunate, mostly when it comes to the instability of nuclear matter. The new rules used by Yukawa are given by the so-called isospin theory of quantum mechanics, the very same introduced by Heisenberg in his theory of nuclear forces and used by Fermi in his theory of beta decay.

The question why a hydrogen nucleus - a proton - is physically stable in spite of the fact that it does not have a neutron in its structure, still remained. Until it was realized that the Geiger-Marsden type of experiments, which led to the notion of nuclear atom, carry still another information with them. Speculating upon the relation between the types of fundamental forces and the structure of matter in which these forces are involved, the conclusion was reached that the nuclear forces do not affect the electrons. Therefore the high energy electrons can be used as 'probes' of the nucleus of hydrogen atom, in Geiger-Marsden type of experiments. This fact opened the door of a new world: that of the overwhelming richness of the structures of nuclear matter. Yukawa theory would touch only a side of the physical explanation of this richness, by associating the forces in this realm with its structural members.

The key point of Yukawa's theory, i.e. the Klein-Gordon equation, like the Helmholtz equation for that matter, is obtainable through a stationary principle, used successfully by Schrödinger in his foundations of the wave mechanics (Schrödinger, 1928, 1933). The Skyrmions came into existence along this line of thought (for a short history and logic see Skyrme, 1988). They are a particular form of solitons associated with a stationary principle, and capable to explain, at least qualitatively, many aspects of the structure of nuclei. This soliton is given by the so-called Skyrme ansatz for the form of solutions of the partial differential equation coming out from the stationary principle. It is this ansatz that makes use of the isotopic spin formalism. The story can continue for a long while with details about the structure of the nucleus. However, let us cut it short here, in order to formulate our actual concern, for we have all the elements in place in order to do it.

The problem represented by the Bohr moment of science, can be formulated approximately like this: from a classical dynamical point of view we have a dynamical model for the atomic structure - the planetary model - under the constraints of some new experimental facts, brought about by the idea that the light should be included into this dynamics. At that moment the model had to offer some reasons in order that those new experimental facts should be properly understood. One can conclude that our spirit reacted by denying the classical way of thinking. Our question is: was it right in doing so? In hindsight, everyone will answer affirmatively, based mostly on the theories of nuclear matter. However, among these theories, the Skyrme theory gives us reasons to 
believe that our spirit was rather inconsistent with itself along its way, in the sense that it has not used properly the concepts at its disposal. The Skyrme theory is, in our opinion, a sign that the classical model of the atom should be reconsidered, and by a proper use of those concepts at that. Our conclusion is sustained in this book, by the analysis of the known Manton geometrization of the theory of Skyrme (Manton, 1987), whereby the concepts of deformations and stresses are (re)considered from a little different angle than the usual ones. This amounts to say that the atomic nucleus does behave like the Sun after all!

The situation of Skyrme theory seems today to have taken the course of any other physical theories, in particular the very ones that led to the concepts related to atom quantization at the beginning of the century past. The issue is no better illustrated than by the words of Edward Witten, extracted from one of the works that have inspired many, if not all, of the ideas of bringing in actuality and generalizing Skyrme theory (Witten, 1976):

Our work raises some obvious questions. Why do these solutions exist, and how can they be generalized? And what relevance do they have for understanding the quantum theory? The answer to these questions we do not know.

These words are indeed best suited for a further clarification of our task here. Specifically, everybody seems to be focused nowadays in the italicized part of Witten's questions: how to generalize. This offers, indeed, a wide field for speculations, appropriate for everyone's intellectual exercise, but leaves uncovered the essential part of the questions raised by Witten. Well, the present work is dedicated to answering them, for the special case of Skyrme theory. In particular, we are here focused in justifying two main hot questions whose answers seem to be swamped into the affluent invasion of works of generalization. The first of these is very specific: why the Skyrme ansatz for the nuclear matter?! It is clearly logical when it comes to considering the particle structure of the nuclear matter. However, is it natural? And if yes, what is its physical foundation? The second is the one explicitly formulated by Witten himself, namely if the skyrmions have indeed relevance for quantization. Our straight answer is that actually there could be no quantization without skyrmions.

In the light of these questions, and their answers as provided in this work, of course, we must say that we are still forced to hang on to the classical planetary model of the atom, at the very least in order to define what seems to be understood by 'natural'. Intuitively assimilable, this model proves to be also theoretically feasible! Come to think of it: one recognizes, on occasions, that there is hardly anything left today from the old 'natural philosophy', the one founded by Newton, and laying at the base of the today's speculative science. The present work brings an amendment to this statement: it is true, indeed, as long as we don't consider the Skyrme theory part of that speculative science! But that seems to be virtually impossible... 
With Skyrme theory, an ubiquitous tendency in the evolution of speculative science seems to be confirmed once again. It appears indeed, that the whole positive science evolves specifically toward explanation of issues left open by Newton himself. And the Skyrme theory fills in for one of the most important issues of Newtonian theory, the definition of force, dealt with incompletely in the classical natural philosophy. Indeed the classical definition of the force is tarnished by a limited possibility of geometrization, which in turn is almost exclusively based upon the idea of material point. While this idea certainly seems to be perfectly suited to task, the tehnological rush enforced a limited concept of material point: that of position in space. This limitation was one of the main original sins of the classical natural philosophy, inherited through its very roots.

Indeed, the Newtonian theory is based on the geometry of motion of celestial bodies, and this is why it was born as a dynamical theory. One might say that, from the original Copernican image that helped in establishing the classical science, only the planets of the solar system were positively contributing to that science. The fixed stars of the Copernican model, although obvious on the canopy to our sense of sight, did not contribute as they should, in order to establish a proper statics for the Newtonian synthesis. That happened because at that historical moment our tehnological focus was on forces. So the theory of light did not have any consequence upon the construction of Newtonian foundations of science. Rather on the contrary: when the time came for the explanation of the light phenomenon through force, in a classical manner, the absence of matter, which is the essential condition of the existence of Newtonian force, was a decisive factor. But then, as today in fact, the fault was not found in the definition of force, but in our concept of light, which was brought to bear material characteristics. Well, the Skyrme theory allows us to correct the vision, inasmuch as it gives us the possibility of improving on the very concept of force, starting exactly from the point where it was left by Newton himself.

In order to give a general idea of what is actually missing, let us see briefly what the Newtonian geometrization left aside by disregarding the fixed stars from the Copernican model. This is obvious from the cosmogonic attempts of Newton himself, which are transparent in his letters to Bishop Bentley (Bentley, 1838, 1842). The only thing that Newton realized, concerning the light in the present association, of course, is the fact that the Sun and stars - the ones producing light - are of the same (physical) nature. This is the starting point of his cosmogony. The rest is inference based on the force he invented, and which, by the way, is not too much different from the today's cosmogony (Weinberg, 1993) that tries to eliminate the force in favour of the allegedly comprehensive, but still quite vague concept of 'interaction'.

Now, should Newton accept the light among his foundational facts, along with the Kepler geometrical synthesis for the motion of planets, he would 
have noticed an important aspect of light, that can go directly to the foundations of science. Indeed, a star is way closer to the condition of material point than the Sun, for this last one has sizable dimensions, apparent to our eyes. Yet in the calculation of the force according to the Newtonian recipe, the Sun is used as a material point, on a par with the remote stars, we might say. The conclusions here are twofold: first the light, as we see it, transcends the scale of time and space, for in the time of Newton it has been recognized explicitly that the stars are way farther than the Sun. The remote stars should be extended bodies just like the Sun, a fact which Newton recognized explicitly. But that was all for him: he couldn't see further the fact that the light as we perceive it is a natural expression of the manifestation of a material point by its variable space expanse, which depends on the point of view. Consequently this variable expanse is not at all obvious in the expression of force of the classical natural philosophy. Thus the force remained subordinate to the concept of material point as a position in space, while the light remained at the mercy of the future electromagnetic theory, that succeeded indeed in explaining it, although only partially, by forces. This is a significant gap which, as Skyrme surely realized, troubled very much Lord Kelvin, who inspired him. As we see it though, only Skyrme theory is able indeed to fill the gap, if we refer it to its proper place in the system of natural philosophy.

As one can guess from this short overview, the present book intends to cover all of the aspects of physical thinking reviewed by Skyrme himself on the occasion of his recollection of history related to skyrmions (Skyrme, 1988). Only, we have to add over what he already said, that we go way back to Newton, from whom everything took rise. Stopping to $19^{\text {th }}$ century natural philosophy for inspiration should not be particularly edifying, inasmuch as that philosophy is pervaded by the advent of technology. The case of Lord Kelvin plainly illustrates what we mean by this statement. Indeed, although "very reluctant to accept the idea of infinitely rigid point-like atoms", Kelvin was unable to even place his own great contributions in the natural order of physical thinking, which they plainly deserve. Skyrme does not appear to realize that the reason for this was the very obstinacy of Kelvin with the technology: "I can never satisfy myself until I can make a mechanical model of a thing. If I can make a mechanical model I can understand it." When "later in his life he came to accept Maxwell's theory" and to abandon his own ideas, he felt like "his life had been a failure".

Newton was the one and only who realized that there is a difference between the technology and science per se, when he said: “... all the difficulty of philosophy seems to consist in this: from the phænomena of motions to investigate the forces of nature, and then from these forces to demonstrate the other phænomena..." (Newton, 1995, Preface; our Italics). The genius of Newton saw the necessity of "forces of nature" in order to substitute for the lack of permanence of our life in the Nature. The technology only offers the delusion 
of "the phænomena of motions" directly, thus eliminating that substitute which happens to be essential. Fortunately the Skyrme's idea seems to bring us back to that essential ingredient...

\section{Conclusions}

The following are the key conclusions of this paper:

i) Classical Mechanics of Newton become fundamental in the development of new modern physics theories (Quantum Mechanics, Fields Theories, etc);

ii) Skyrme Theories are contained in the Newtonian Theory taking into consideration the scale resolution in sens of Mandelbrot;

iii) Even though Skyrme Theories describe matter behaviors at a nucleonic scale, it does not disclaim the classical Newtonian concepts.

\section{REFERENCES}

Bentley R., Works, Vol. III, Francis Macpherson, London (1838).

Bentley R., Correspondence, Vol. I, John Murray, London (1842).

Beyer R.T., Foundations of Nuclear Physics, Dover Publications, New York (1949).

Bohr N., On the Constitution of Atoms and Molecules, The Philosophical Magazine, Vol. 26, pp. 1-24 (1913).

Brink D.M., Nuclear Forces, Pergamon Press, Oxford (1965).

Einstein A., Concerning an Heuristic Point of View Toward the Emission and Transformation of Light, American Journal of Physics, Vol. 33 (1965), English translation of the original from Annalen der Physik, Vol. 17 (1905).

Gamow G., Zur Quantentheorie des Atomkernes, Zeitschrift fur Physik, Vol. 51, pp. 204-212 (1928).

Lewis G.N., The Conservation of Photons, Nature, Vol. 118, pp. 874-875 (1926).

Mandelbrot B.B., The Fractal Geometry of Nature, W. H. Freeman and Co., San Fracisco (1982).

Manton N.S., Geometry of Skyrmions, Communications in Mathematical Physics, Vol. 111, pp. 469-478 (1987).

Newton I., The Principia, Prometheus Books, Amherst, New York (1995).

Pauli W., General Principles of Quantum Mechanics, Springer-Verlag, Berlin (1980).

Rutherford E., The Scattering of $\alpha$ and $\beta$ Particles by Matter and the Structure of the Atom, The Philosophical Magazine, Vol. 21, pp. 669-688 (1911).

Schrödinger E., Collected Papers on Wave Mechanics, Blackie \& Son Limited, London (1928).

Schrödinger E., Mémoirs Sur La Mécanique Ondulatoire, Félix Alcan, Paris (1933).

Seeliger H., Uber das Newton'sche Gravitationsgesetz, Astronomische Nachrichten, Vol. 137, pp. 129-136 (1895).

Skyrme T.H.R., The Origins of Skyrmions, International Journal of Modern Physics, Vol. A3, 2745-2751 (1988). 
Thomson J.J., Electricity and Matter, Charles Scribner's Sons, New York (1907).

Weinberg S., The First Three Minutes, Basic Books (1993).

Witten E.B., Some Exact Multi-Instanton Solutions of Classical Yang-Mills Theory, HUTP-76 A172b, Physical Review Letters, Vol. 38, pp. 121-124 (1976).

Yukawa H., On the Interaction of Elementary Particles, Progress of Theoretical Physics (Japan), Vol. 17, p. 48 (1935).

\section{DE LA FILOZOFIA CLASICĂ NEWTONIANĂ LA SKYRMION. O SCURTĂ ISTORIE}

\section{(Rezumat)}

Această lucrare prezintă o scurtă istorie a evoluției modelelor teoretice în fizică, de la Principiile Matematice ale Filozofiei Naturale a lui Newton la skyrmionii lui Skyrme din Modelele Nucleonice. În aceste condiții Teoria Newtoniană devine fundamentală în orice altă construcție teoretică a fizicii moderne (ex. Mecanica Cuantică, Teoria Câmpurilor, etc.). 